\title{
Hemorragia peripapilar em pacientes com glaucoma e sua relação com o tronco vascular retiniano
}

\author{
Association between optic disc hemorrhages and the position \\ of central retinal vessels
}

\author{
Sérgio Henrique Teixeira ${ }^{1}$ \\ Paulo Aug'usto de Arruda Mello 2 \\ AugiustoParanhos Júnior ${ }^{3}$
}

\begin{tabular}{l} 
RESUMO \\
\hline Objetivos: Avaliar a correlação entre as hemorragias peripapilares (HPPs) \\
e a posição do tronco vascular na cabeça do nervo óptico. Métodos: Reti- \\
nografias de 1.830 pacientes foram revisadas. A posição do tronco vascular \\
retiniano (TVR) foi classificada de acordo com sua localização no hemis- \\
fério superior ou inferior e sua posição relativa através da razão entre a \\
distância entre o TVR e o ponto mais inferior do disco óptico e o diâmetro \\
vertical do disco óptico (razão tronco/disco). Pacientes da mesma popu- \\
lação, com glaucoma e sem HPPs foram aleatoriamente selecionados para \\
comparação. Resultados: Dos 1.830 pacientes, 46 apresentaram HPPs. \\
Foram detectadas 53 hemorragias em 41 pacientes com glaucoma ou \\
suspeitos de glaucoma, principalmente na região temporal inferior (47,2\%). \\
Observou-se concordância negativa entre a localização superior ou in- \\
ferior do TVR e das HPPs (Kappa= -0,34). Considerando a posição \\
relativa do TVR em relação ao diâmetro vertical do disco óptico (razão \\
tronco/disco), encontramos melhor correlação (valores médios da razão \\
tronco/disco: 0,450-hemorragias superiores e 0,510-hemorragias inferio- \\
res; p=0,027). No grupo com HPPs, observamos porcentagem significati- \\
vamente maior de pacientes com glaucoma de pressão normal (GPN) se \\
comparado ao grupo sem hemorragia (p=0,009). Conclusão: HPPs foram \\
mais comuns no hemisfério oposto ao da emergência do TVR. Observamos \\
maior prevalência de GPN nos pacientes com hemorragias que nos sem \\
hemorragia.
\end{tabular}

Descritores: Nervo óptico, hemorragia retiniana/etiologia; Vasos retinianos; Glaucoma
Trabalho realizado no Departamento de Oftalmologia da Universidade Federal de São Paulo - UNIFESP - São Paulo (SP) - Brasil.

${ }^{1}$ Colaborador do Setor de Glaucoma da Universidade Federal de São Paulo - UNIFESP - São Paulo (SP) Brasil.

${ }^{2}$ Professor Adjunto e Orientador do Programa de Pósgraduação da UNIFESP - São Paulo (SP) - Brasil.

${ }^{3}$ Livre Docente, Professor do Programa de Pós-graduação da UNIFESP - São Paulo (SP) - Brasil; Gerente de Pesquisa Clínica do Instituto Israelita de Ensino e Pesquisa Albert Einstein - IIEPAE - São Paulo (SP) Brasil.

Endereço para correspondência: Sérgio Henrique Teixeira. Av. Indianópolis, 1797 - São Paulo (SP) CEP 04063-003

E-mail: sergio@oftalmo.epm.br

Recebido para publicação em 10.03.2008 Aprovação em 23.05.2008 \begin{tabular}{l} 
INTRODUÇÃo \\
\hline A presença de hemorragias peripapilares (HPPs) é um sinal importante \\
tanto no diagnóstico quanto no acompanhamento do glaucoma. Por serem \\
bastante específicas, facilitam o diagnóstico nos casos em que os outros \\
exames estão negativos ou inconclusivos, como no glaucoma pré-perimé- \\
trico por exemplo ${ }^{(1-4)}$. Mesmo sendo muito características do glaucoma, \\
podem surgir em outras situações como: oclusões de veia central da retina, \\
edema de papila, descolamento de vítreo posterior (DVP), diabetes, entre \\
outras ${ }^{(5-8)}$. São importantes no acompanhamento porque geralmente indi- \\
cam que a doença está mal controlada: sabe-se que estão associadas à \\
progressão do dano glaucomatoso ${ }^{(5,9-12)}$, e que HPPs de repetição estão mais \\
associadas à progressão que episódios isolados ${ }^{(10)}$ e ainda que a diminuição \\
da pressão intra-ocular diminui a incidência de hemorragias ${ }^{(13)}$. \\
Apesar de sua importância clínica, a patogênese das HPPs não está
\end{tabular} 
totalmente esclarecida e as principais teorias dizem respeito a fatores mecânicos (como a oclusão de pequenos vasos pela pressão intra-ocular ou por movimentações da lâmina crivosa $)^{(1,14)}$ e fatores vasculares com danos nas paredes capilares ${ }^{(5)}$ podendo até sofrer influências de fatores ligados à coagulação ${ }^{(15)}$. Com relação à lâmina crivosa, foi proposto que a proximidade do tronco vascular retiniano (TVR) poderia exercer um efeito estabilizador e proteger a região adjacente do dano glaucomatoso ${ }^{(1)}$. Já foi demonstrado que a presença de atrofia zona beta e a presença de atrofia localizada na camada de fibras nervosas (sinal de Hoyt) se correlacionam negativamente com a localização da emergência do TVR na cabeça do nervo óptico ${ }^{(16-17)}$. De maneira semelhante, também já se demonstrou a associação entre as HPPs e a presença do sinal de Hoyt e atrofias peripapilares ${ }^{(18-19)}$. Até o momento, desconhecemos algum estudo avaliando a relação entre as localizações do TVR e das HPPs.

No presente estudo pretendemos avaliar a correlação entre as HPPs e a posição da emergência do TVR na cabeça do nervo óptico.

\section{MÉTODOS}

Retinografias estereoscópicas de 1.830 pacientes com glaucoma (Gl), suspeita de glaucoma (SGl) ou outras moléstias retinianas foram revisadas individualmente em busca de hemorragias peripapilares. Os exames foram realizados no período de janeiro de 2006 a setembro de 2007 com um retinógrafo digital modelo CR-DGi - Canon (Lake Success, NY, EUA).

As HPPs foram avaliadas quanto a sua localização, freqüência e presença de fatores associados como edema de papila, sinal de Hoyt adjacente, hemorragia pré-retiniana ou outras alterações fundoscópicas.

Com base em um modelo padronizado (Figura 1), as HPPs foram classificadas por um especialista em glaucoma de acordo com a sua localização em: temporal, temporal superior, temporal inferior, inferior, nasal inferior, nasal e nasal superior.

Os prontuários e os exames dos pacientes foram analisados retrospectivamente para determinar as características clínicas e o diagnóstico no momento da hemorragia.

O glaucoma foi diagnosticado quando o paciente apresentava: 1- campo visual alterado com defeito típico compatível com alteração no nervo óptico ou 2- defeito anatômico típico (como sinal de Hoyt, razão escavação/disco acima de 0,7, defeito localizado na rima neural ou assimetria de escavação) ${ }^{(20)}$. Pacientes com glaucoma de ângulo aberto e pressão intra-ocular abaixo de $21 \mathrm{mmHg}$ (em todas as visitas, na ausência de medicação antiglaucomatosa) foram diagnosticados como portadores de glaucoma de pressão normal. Se o paciente apresentava hemorragia peripapilar, ausência de sinais de oclusões venosas ou edema de papila, ausência de sinais ou sintomas de DVP (hemorragia pré-retiniana, floaters, etc) e algum fator de risco para glaucoma (PIO acima de $21 \mathrm{mmHg}$, razão escavação/disco entre 0,5 e 0,7 ou história familiar), foi classificado como suspeito de glaucoma.

Um grupo de pacientes do mesmo banco de dados de retinografias, com glaucoma e sem hemorragias detectadas, foi aleatoriamente selecionado para comparação de dados epidemiológicos (idade, sexo, raça) e oftalmológicos (diagnóstico, índice "mean deviation" da campimetria computadorizada e classificação do dano no nervo óptico). Os exames de campimetria foram realizados com o "Humphrey Field Analyzer" modelo 750 (Carl Zeiss Meditech, Dublin, CA, EUA), na estratégia SITA 24-2 branco sobre branco, em todos os pacientes e a classificação do defeito no nervo óptico foi feita com um escore de 1 a 10 proposto por Speath ${ }^{(21)}$ (DDLS"Disk Damage Likelihood Stage"). Para esta comparação, foram excluídos os pacientes que possuíam catarata ou outras opacidades de meios e cirurgia refrativa prévia.

Dentre pacientes com glaucoma, foram selecionados aqueles cuja cabeça do nervo óptico apresentava uma emergência identificável do TVR. A posição do TVR foi classificada de acordo com sua localização no hemisfério superior ou inferior com auxilio de um software de medição acoplado ao retinógrafo digital (Figura 2). Para estimar a posição relativa do TVR, dividimos a distância entre o TVR e o ponto mais inferior do disco óptico pelo diâmetro vertical do disco óptico (razão tronco/disco). Dessa forma, indivíduos com uma razão tronco/disco acima de 0,5 possuíam um TVR emergin-

\section{Localização das hemorragias}

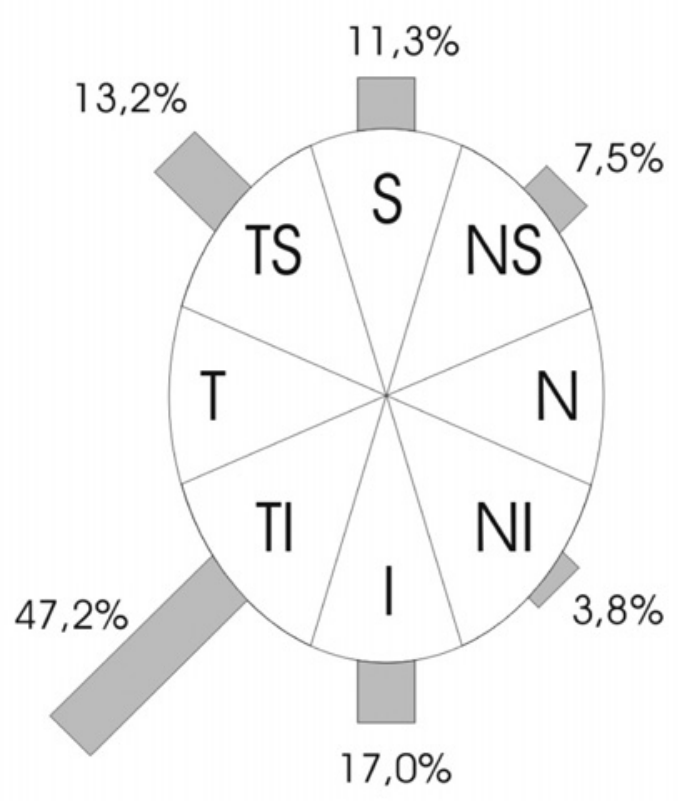

Figura 1 - Hemorragias peripapilares quanto à localização nos pacientes com glaucoma e suspeitos de glaucoma. As hemorragias do olho direito e olho esquerdo foram agrupadas no gráfico e a representação do olho esquerdo foi adicionada de maneira espelhada à do olho direito. $\mathrm{S}=$ superior; $\mathrm{TS}=$ temporal superior; $\mathrm{T}=$ temporal; $\mathrm{Tl}=$ temporal inferior; I= inferior; $\mathrm{Nl}=$ nasal inferior; $\mathrm{N}=$ nasal; $\mathrm{NS}=$ nasal superior. 
do em uma posição deslocada superiormente ao longo do diâmetro vertical do disco óptico.

A concordância entre a localização superior ou inferior do TVR e a localização das HPPs foi avaliada pelo teste Kappa. A diferença entre a posição relativa do TVR (razão tronco/ disco), nos pacientes com hemorragias superiores e inferiores, foi avaliada através do teste- $t$. Os dados foram organizados em planilhas do Microsoft Excel e a avaliação estatística com o software NCSS/PASS.

\section{RESULTADOS}

Dos 1.830 pacientes, 46 apresentaram HPPs. Destes, cinco apresentavam sinais e história de edema de papila ou oclusão venosa e foram excluídos da análise.

Foram detectadas 53 HPPs em 41 pacientes com glaucoma ou suspeitos de glaucoma, principalmente na região temporal inferior e temporal superior (Figura 1).

Dos pacientes com glaucoma ou suspeitos de glaucoma, nove apresentaram hemorragias recorrentes (em mais de uma retinografia), sendo que em cinco pacientes elas foram detectadas no mesmo exame, em regiões diferentes da cabeça do nervo óptico ou no outro olho.

A média da idade dos pacientes com HPPs foi de 63,6 anos $(42-87$; $\mathrm{DP}=11,7)$, sem diferença significativa entre homens e mulheres $(62,6$ e 64,3 respectivamente, $p=0,40)$.

Trinta e quatro pacientes apresentavam diagnóstico de glaucoma por ocasião das HPPs.

Dos pacientes com glaucoma 32 apresentavam a emergência do TVR identificável. Através do teste de Kappa, demonstrou-se uma concordância negativa entre a localização do TVR e as HPPs (Kappa $=-0,34$ ) (Tabela 1). Levando-se em consideração a posição relativa do TVR em relação ao diâmetro vertical do disco óptico (razão tronco/disco), encontramos uma melhor correlação $(-0,39)$, com valores médios da razão tronco/disco de 0,450 para as hemorragias superiores e 0,510 para as hemorragias inferiores (diferença $=-0,060$; $\mathrm{IC}=-0,113$ a $-0,007 ; \mathrm{p}=0,027$ ) (Figura 3 ).

Uma extração aleatória de pacientes com glaucoma da mesma população apresentou uma porcentagem significativamente menor de pacientes com GPN que no grupo com hemorragia ( $\mathrm{p}<0,01$ - qui-quadrado) e não apresentou diferença estatisticamente significante quanto à idade, sexo, raça ou outros parâmetros oftalmológicos (Tabela 2).

\section{DISCUSSÃO}

As HPPs são muito específicas do glaucoma, mas não são patognomônicas e podem ocorrer em outras situações ${ }^{(6-8)}$. Neste estudo, dos 46 pacientes com hemorragias, aproximadamente $10 \%$ apresentavam diagnóstico de oclusão venosa, descolamento de vítreo posterior ou edema de papila. Nos pacientes suspeitos de glaucoma, as HPPs podem ser um

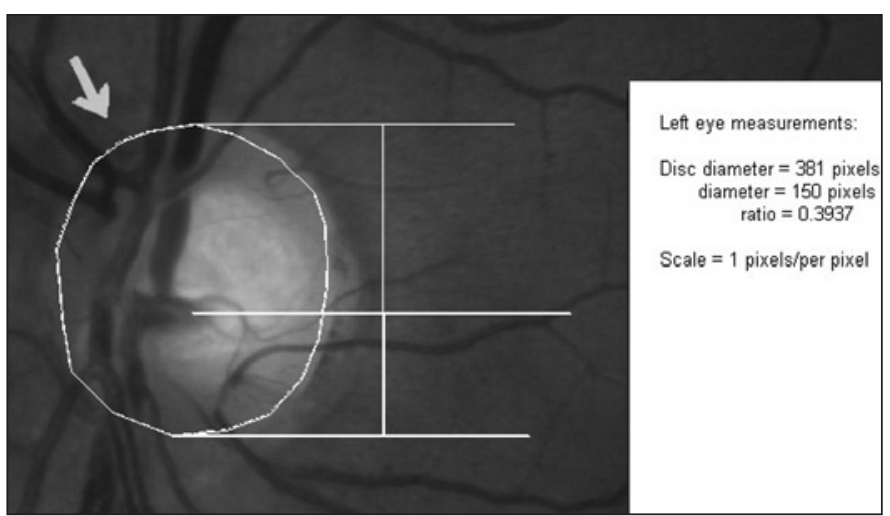

Figura 2 - Exemplo da classificação da localização do tronco vascular na cabeça do nervo óptico. 0 primeiro diâmetro ("diameter") refere-se ao diâmetro vertical do nervo óptico e o segundo diâmetro à distância entre a porção mais inferior do nervo e a posição do tronco vascular. A razão ("ratio") entre o segundo e o primeiro diâmetro (razão tronco/ disco) fornece a posição relativa do tronco (superior= razão acima de $0,5$ e inferior= razão abaixo de 0,5$)$. A seta indica pequena hemorragia na região nasal superior.

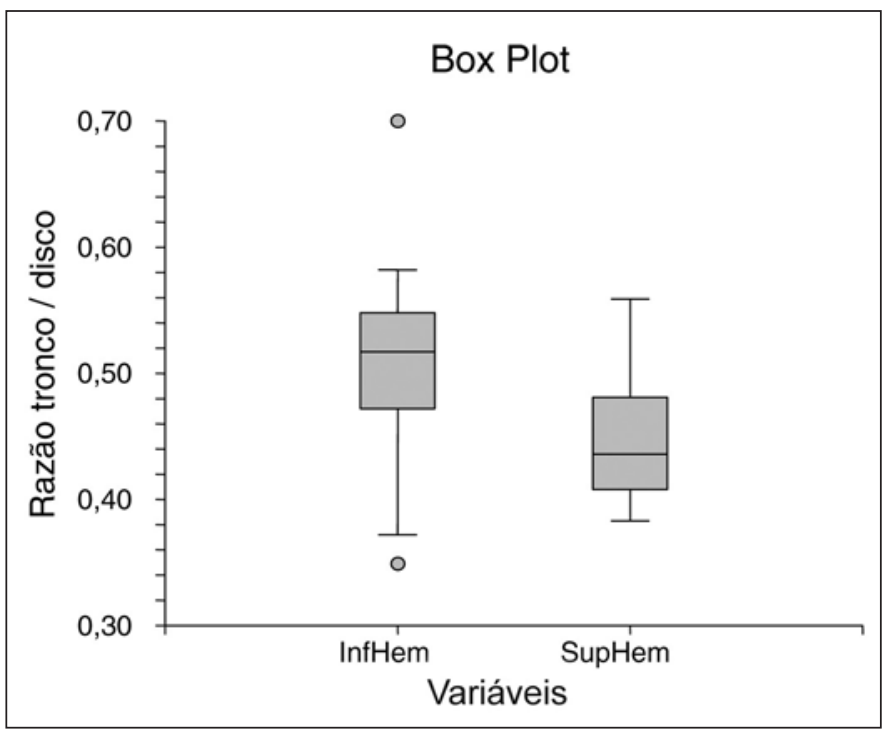

Figura 3 - Comparação da posição relativa do tronco vascular retiniano dos pacientes com hemorragias superiores e inferiores. NCSS boxplot - gráfico para estatística do teste-t de variância iguais, $p=0,027$; Wilcoxson $\mathrm{p}=\mathbf{0 , 0 1 3}$; InfHem= pacientes com hemorragias peripapilares inferiores; SupHem= pacientes com hemorragias peripapilares superiores.

primeiro sinal do desenvolvimento da doença $\mathrm{a}^{(4)}$ ou podem ter uma etiologia ainda não esclarecida. Por isso, mesmo não sendo patognomônicas, são sinais preciosos no diagnóstico e no acompanhamento do glaucoma.

HPPs são mais comuns na região temporal próxima aos pólos inferiores e superiores da cabeça do nervo óptico. A região mais freqüente é a temporal inferior ${ }^{(1,4-5,8,20)}$, fato que foi confirmado por este estudo. O oftalmologista deve, no entanto, ficar atento à presença de HPPs em áreas não usuais como nasal superior e inferior ( 7,5 e 3,8\% nesta série), pois estas são freqüentemente menos exuberantes que as tempo- 


\begin{tabular}{|lccc|}
\hline Tabela 1. Diagnósticos dos pacientes com hemorragias peripa- & $\begin{array}{c}\text { pilares } \\
\text { pilar }\end{array}$ & \multicolumn{4}{c}{ Tronco vascular } \\
\cline { 2 - 4 } Hemorragia & Superior & Inferior & Total \\
Superior & 2 & 8 & 10 \\
Inferior & 15 & 7 & 22 \\
Total & 17 & 15 & 32 \\
Kappa $=-0,34$ & & & \\
\hline
\end{tabular}

\begin{tabular}{|c|c|c|c|}
\hline & $\begin{array}{c}\text { Com hemorragia } \\
\qquad(n=31)\end{array}$ & $\begin{array}{c}\text { Sem hemorragia } \\
(n=31)\end{array}$ & $p$ \\
\hline \multicolumn{4}{|l|}{ Diagnóstico } \\
\hline GPN & 17 & 6 & $0,009^{\#}$ \\
\hline Outros & 14 & 25 & \\
\hline GCS & 7 & 18 & \\
\hline GCAE & 3 & 6 & \\
\hline GSEC & 1 & 0 & \\
\hline GP & 3 & 1 & \\
\hline \multicolumn{4}{|l|}{ Sexo } \\
\hline Masculino & 15 & 12 & $0,744^{\#}$ \\
\hline Feminino & 16 & 19 & \\
\hline Idade & 63,6 & 58,9 & $0,247^{*}$ \\
\hline \multicolumn{4}{|l|}{ Olho } \\
\hline Direito & 9 & 15 & $0,294^{\#}$ \\
\hline Esquerdo & 22 & 16 & \\
\hline \multicolumn{4}{|l|}{ Raça } \\
\hline Brancos & 22 & 18 & $0,569^{\#}$ \\
\hline Outros & 9 & 13 & \\
\hline Orientais & 9 & 10 & \\
\hline Negros & 0 & 3 & \\
\hline \multicolumn{4}{|l|}{ Campimetria } \\
\hline$M D$ & $-3,0$ & $-5,8$ & $0,050^{*}$ \\
\hline PSD & 3,4 & 4,8 & $0,142^{*}$ \\
\hline \multicolumn{4}{|l|}{ Nervo óptico } \\
\hline DDRS & 4,0 & 4,0 & $0,213^{+}$ \\
\hline Paquimetria & 543,4 & 537,1 & $0,539^{*}$ \\
\hline \multicolumn{4}{|c|}{$\begin{array}{l}\text { n= número de pacientes; GCS= glaucoma crônico símples; GPN= glaucoma de } \\
\text { pressão normal; GCAE= glaucoma crônico de ângulo estreito; GSEC= glaucoma } \\
\text { secundário; GP= glaucoma pigmentar; } \mathrm{MD}=\text { "mean deviation"; } \mathrm{PSD}=\text { "pattern } \\
\text { standard deviation"; DDRS= "Disk Damage Likelihood Stage"; "= teste qui } \\
\text { quadrado; "= teste t bicaudal; += teste de Wilcoxson }\end{array}$} \\
\hline
\end{tabular}

rais $^{(5)}$ (Figura 2). Essa informação é fundamental, pois apenas o exame minucioso da cabeça do nervo óptico pode detectar algumas hemorragias e a não detecção das mesmas pode significar um diagnóstico mais tardio do glaucoma ou da progressão do dano glaucomatoso.

Até onde temos conhecimento, a associação negativa entre a emergência do TVR na lâmina crivosa e a presença de HPPs no mesmo hemisfério (superior ou inferior) da cabeça do nervo óptico foi descrita pela primeira vez neste estudo. A associação negativa indica que existe uma correlação entre possuir hemorragia em um hemisfério e tronco vascular em outro, assim, na amostra estudada, os pacientes que apresen- tavam HPPs em um hemisfério mais freqüentemente apresentavam a emergência do TVR no outro. Este achado pode significar que existe um papel protetor do tronco vascular na rima nervosa adjacente ao mesmo como sugerido por outros estudos $^{(16-17)}$. As limitações deste estudo envolvem o relativamente pequeno número de pacientes com o tronco vascular claramente identificável (parcialmente justificável devido à raridade das HPPs). Isso gerou um intervalo de confiança relativamente amplo e deve ser levado em conta na interpretação dos resultados. Apesar de estatisticamente significante, a correlação moderada encontrada indica que outros fatores têm forte influência na localização das HPPs.

O método escolhido para classificar a posição relativa do TVR (a razão tronco/disco) vai além da análise dicotomizada da posição (superior e inferior) e não necessita levar em conta o tamanho do nervo óptico, já que lida com uma razão entre dois diâmetros. Como desvantagem, ele não possibilita uma avaliação de uma possível influência da real distância entre as HPPs e o TVR, o que teria que levar em conta fatores como magnificação ocular e do retinógrafo e o tamanho do nervo óptico. Apesar disso é um método simples e portanto facilmente reprodutível. Além disso, o posicionamento ao longo do diâmetro vertical do disco foi escolhido devido à separação hemisférica das fibras nervosas na retina.

Esta série encontrou um maior número de pacientes com glaucoma de pressão normal no grupo com HPPs que no grupo sem HPPs, concordando com estudos que relataram maior prevalência de hemorragias em pacientes com glaucoma de pressões baixas ${ }^{(8,20,22)}$. Isso pode auxiliar o diagnóstico do glaucoma, principalmente em se tratando de populações onde coexistem diferentes grupos étnicos como no presente estudo. Como já foi dito, as HPPs são muito específicas e podem ajudar no diagnóstico do glaucoma, do tipo de glaucoma e de um eventual descontrole clínico da doença.

\section{CONCLUSÃO}

Demonstramos que as hemorragias peripapilares são mais comuns no hemisfério oposto ao da emergência do tronco vascular retiniano. Observamos também uma maior prevalência de GPN nos pacientes com hemorragias comparados aos sem hemorragia.

\section{ABSTRACT}

Purpose: To investigate the correlation between optic disc hemorrhages (ODHs) and the position of the central retinal vessel trunk (RVT) in patients with glaucoma. Methods: Fundus photographs of 1,830 participants were reviewed. The exit position of the central retinal vessel trunk was classified as being in the superior or inferior hemisphere of the optic disc and the relative vertical position was expressed as a fraction of the disc diameter (ratio between the position of the trunk 
and the diameter of the disk). A group of patients from the same population, but without ODHs, was randomly selected and compared with the patients with ODHs. Results: Among the 1,830 patients, 53 hemorrhages were detected in 41 patients with glaucoma or suspected glaucoma. We found an inverse relationship between the inferior/superior position of the central RVT and the inferior/superior position of the hemorrhages $(\mathrm{Kappa}=-0.34)$. Using the trunk/disc ratio, we found that superior ODHs correlated with inferior RVTs (trunk/ disc ratio, 0.450; $\mathrm{SD}=0.070)$ and inferior $\mathrm{ODHs}$ correlated with superior RVTs (trunk/disc ratio, 0.510; $\mathrm{SD}=0.054)(\mathrm{p}=0.027)$. The number of patients with normal tension glaucoma was significantly higher among patients with ODHs than among those without ODHs $(\mathrm{p}=0.009)$. Conclusion: Our patients had a negative correlation between the position of central RVT and the location of the ODHs. Normal tension glaucoma was more prevalent among patients with ODHs than among those without it.

Keywords: Optic nerve; Retinal hemorrhage/etiology; Retinal vessels; Glaucoma

\section{REFERÊNCIAS}

1. Jonas JB, Budde WM, Panda-Jonas S. Ophthalmoscopic evaluation of the optic nerve head. Surv Ophthalmol. 1999;43(4):293-320. Review.

2. Drance SM, Begg IS. Sector haemorrhage - a probable acute ischaemic disc change in chronic simple glaucoma. Can J Ophthalmol. 1970;5(2):137-41.

3. Susanna R, Drance SM, Douglas GR. Disc hemorrhages in patients with elevated intraocular pressure. Occurrence with and without visual field changes. Arch Ophthalmol. 1979;97(2):284-5.

4. Budenz DL, Anderson DR, Feuer WJ, Beiser JA, Schiffman J, Parrish RK $2^{\text {nd }}$, Piltz-Seymour JR, Gordon MO, Kass MA; Ocular Hypertension Treatment Study Group. Detection and prognostic significance of optic disc hemorrhages during the Ocular Hypertension Treatment Study. Ophthalmology. 2006; 113(12):2137-43.

5. Sonnsjö B, Dokmo Y, Krakau T. Disc haemorrhages, precursors of open angle glaucoma. Prog Retin Eye Res. 2002;21(1):35-56.

6. Bengtsson B. Chronic glaucoma and symptomatic vitreous detachment. Acta Ophthalmol (Copenh). 1986;64(2):152-6.
7. Katz B, Hoyt WF. Intrapapillary and peripapillary hemorrhage in young patients with incomplete posterior vitreous detachment. Signs of vitreopapillary traction. Ophthalmology. 1995;102(2):349-54. Comment in: Ophthalmology. 1995;102(7):1003-4. Ophthalmology. 1996;103(4):543.

8. Yamamoto T, Iwase A, Kawase K, Sawada A, Ishida K. Optic disc hemorrhages detected in a large-scale eye disease screening project. J Glaucoma. 2004;13(5):356-60.

9. Leske MC, Heijl A, Hussein M, Bengtsson B, Hyman L, Komaroff E; Early Manifest Glaucoma Trial Group. Factors for glaucoma progression and the effect of treatment: the early manifest glaucoma trial. Arch Ophthalmol. 2003; 121(1):48-56.

10. Kim SH, Park KH. The relationship between recurrent optic disc hemorrhage and glaucoma progression. Ophthalmology. 2006;113(4):598-602.

11. Ishida K, Yamamoto T, Sugiyama K, Kitazawa Y. Disk hemorrhage is a significantly negative prognostic factor in normal-tension glaucoma. Am J Ophthalmol. 2000;129(6):707-14. Comment in: Am J Ophthalmol. 2000;129 (6):796-7.

12. Siegner SW, Netland PA. Optic disc hemorrhages and progression of glaucoma. Ophthalmology. 1996;103(7):1014-24. Comment in: Ophthalmology. 1997;104(4):566-7. Ophthalmology. 1997;104(2):169.

13. Miyake T, Sawada A, Yamamoto T, Miyake K, Sugiyama K, Kitazawa Y. Incidence of disc hemorrhages in open-angle glaucoma before and after trabeculectomy. J Glaucoma. 2006;15(2):164-71.

14. Quigley HA, Addicks EM, Green WR, Maumenee AE. Optic nerve damage in human glaucoma. II. The site of injury and susceptibility to damage. Arch Ophthalmol. 1981;99(4):635-49.

15. Soares AS, Artes PH, Andreou P, Leblanc RP, Chauhan BC, Nicolela MT. Factors associated with optic disc hemorrhages in glaucoma. Ophthalmology. 2004;111(9):1653-7.

16. Jonas JB, Budde WM, Németh J, Gründler AE, Mistlberger A, Hayler JK. Central retinal vessel trunk exit and location of glaucomatous parapapillary atrophy in glaucoma. Ophthalmology. 2001;108(6):1059-64.

17. Jonas JB, Fernández MC. Shape of the neuroretinal rim and position of the central retinal vessels in glaucoma. Br J Ophthalmol. 1994;78(2):99-102.

18. Ahn JK, Kang JH, Park KH. Correlation between a disc hemorrhage and peripapillary atrophy in glaucoma patients with a unilateral disc hemorrhage. J Glaucoma. 2004;13(1):9-14.

19. Sugiyama K, Tomita G, Kitazawa Y, Onda E, Shinohara H, Park KH. The associations of optic disc hemorrhage with retinal nerve fiber layer defect and peripapillary atrophy in normal-tension glaucoma. Ophthalmology. 1997;104 (11):1926-33.

20. Healey PR, Mitchell P, Smith W, Wang JJ. Optic disc hemorrhages in a population with and without signs of glaucoma. Ophthalmology. 1998;105 (2):216-23.

21. Spaeth GL, Henderer J, Steinmann W. The Disc Damage Likelihood Scale (DDLS): its use in the diagnosis and management of glaucoma. Highlights Ophthalmol. 2003;31:4-19.

22. Kitazawa Y, Shirato S, Yamamoto T. Optic disc hemorrhage in low-tension glaucoma. Ophthalmology. 1986;93(6):853-7. 